\title{
Spectroscopy of the Optical Counterpart to Ser X-1
}

\author{
R. I. Hynes ${ }^{1,2 \star}$ P. A. Charles ${ }^{2}$, L. van Zyl ${ }^{3,4}$, A. Barnes ${ }^{2}$, D. Steeghs ${ }^{5,2}$, \\ K. O’Brien ${ }^{6,7}$, J. Casares ${ }^{8}$ \\ ${ }^{1}$ Astronomy Department, The University of Texas at Austin, 1 University Station C1400, Austin, Texas 78712-0259, USA \\ ${ }^{2}$ Department of Physics and Astronomy, University of Southampton, Southampton, SO17 1BJ, UK \\ ${ }^{3}$ Department of Astrophysics, Oxford University, Keble Road, Oxford, OX1 3RH, UK \\ ${ }^{4}$ Astrophysics Group, School of Chemistry and Physics, Keele University, Keele, Staffordshire, ST5 5BG, UK \\ ${ }^{5}$ Harvard-Smithsonian Center for Astrophysics, 60 Garden Street, MS-67, Cambridge, MA 02138, USA \\ ${ }^{6}$ European Southern Observatory, Casilla 19001, Santiago 19, Chile \\ ${ }^{7}$ School of Physics and Astronomy, University of St Andrews, St Andrews KY16 9SS, UK \\ ${ }^{8}$ Instituto de Astrofísica de Canarias, 38200 La Laguna, Tenerife, Spain
}

Accepted ?. Received ?; in original form?

\begin{abstract}
We present WHT and VLT spectroscopy of MM Ser, the optical counterpart to Ser X1. We deblend the red spectra of the two close stars identified by Wachter (1997) and show that the brighter of the two is responsible for the $\mathrm{H} \alpha$ and $\mathrm{He}$ I emission, hence confirming that this is the true counterpart of the X-ray source. We also identify several He II and N III lines in the blue spectrum. The isolated emission lines are all remarkably narrow, with FWHM $200-300 \mathrm{~km} \mathrm{~s}^{-1}$. The Bowen blend has structure suggesting that the individual components are also narrow. These narrow lines could be from the disc if the binary inclination is quite low, or they could come from a more localised region such as the heated face of the companion star. Several interstellar lines are detected and indicate that the reddening is moderate, and consistent with the neutral hydrogen column density inferred in X-rays.
\end{abstract}

Key words: accretion, accretion discs - binaries: close - stars: individual: MM Ser

\section{INTRODUCTION}

The low-mass X-ray binary (LMXB) Ser X-1 has been known as an X-ray source from the early days of X-ray astronomy. It was discovered in 1965 (Friedman, Byrom \& Chubb 1967). Its optical counterpart has proven more elusive, however. When an accurate $(1$ arcmin) position for the X-ray source became known (Doxsey 1975), Davidsen (1975) suggested that the optical counterpart was an ultraviolet excess object with $B \sim 18.5$. Subsequently Thorstensen, Charles \& Bowyer (1980) used images obtained in better seeing conditions to show that this counterpart was actually two coincident stars (DN and DS) separated by 2.1 arcsec. The southern one, designated MM Ser, was by far the brighter in the ultraviolet. The detection of an optical burst from DS, simultaneously with an X-ray burst (Hackwell et al. 1979), confirmed that the X-ray source was associated with DS. More recently, however, Wachter (1997) has shown that DS is itself two unresolved stars (DSe and DSw), separated by only 1 arcsec. Wachter suggested that the brighter of the two stars, which is bluer, might be the true optical counterpart. Silber (1998) has analysed rapid photometry

\footnotetext{
^ E-mail: rih@astro.as.utexas.edu; Hubble Fellow
}

which supports this, indicating that the brighter of the two stars is significantly variable. No convincing periodicity was found in these data, however.

Spectroscopy of MM Ser was obtained at several epochs, before it was resolved into two stars. Thorstensen et al. (1980) obtained blue spectroscopy of both DS and DN. Both showed $\mathrm{H} \beta$ absorption, but DS in addition had a He II $4686 \AA$ emission line. Cowley, Hutchings \& Crampton (1988) confirmed the presence of narrow He II emission. Their spectrum shows no $\mathrm{H} \beta$ absorption, but possible N III $4640 \AA$ emission. Shahbaz et al. (1996) reported an almost featureless continuum spanning the full optical bandpass, albeit at a very low resolution which would be insensitive to weak emission features. An absorption feature around $5900 \AA$ was attributed to a $\mathrm{G}$ star secondary.

We report on further spectroscopy of MM Ser. Our primary goal was to separate the spectra of DSe and DSw and hence confirm which is the true optical counterpart to Ser X1. Since the most recent published spectroscopy dates from 1988 we also were able to obtain a higher quality spectrum using modern instrumentation and hence study the properties of MM Ser in more detail than previously possible. 


\section{OBSERVATIONS}

\subsection{WHT spectra}

Spectra of MM Ser were obtained from the Observatorio del Roque de los Muchachos on La Palma on the nights of 2001 July 8 and 10 using the $4.2 \mathrm{~m}$ William Herschel Telescope (WHT) with the ISIS dual-arm spectrograph. The seeing was typically $1.0-1.5 \operatorname{arcsec}$ on July 8 and $\sim 0.8 \operatorname{arcsec}$ on July 10.

The slit width was set to 0.83 arcsec. The slit was aligned to pass through the two blended stars (DSe and DSw) identified by Wachter (1997). The blue arm was used with the EEV12 CCD and the R600B grating to yield a resolution of $1.9 \AA$. Note that the blue spectrum is heavily vignetted at either end; the useful coverage spans 3720 $5260 \AA$. On July 8 the red arm was used with the TEK4 $\mathrm{CCD}$ and the R600R grating for a resolution of $1.5 \AA$. On July 10 the R316R grating was instead used giving a resolution of $3.1 \AA$. A log of the spectra obtained of MM Ser is given in Table 1

Basic image processing (bias correction and flat fielding) was done using standard IRAF ${ }^{1}$ techniques. Unfortunately no suitable red arm flat field was obtained on July 10, so it was necessary to use the July 8 one which was obtained with a different grating.

Initially a combined spectrum of both stars was extracted using the IRAF implementation of optimal extraction (Horne 1986; Marsh 1989). As there are actually two blended stars at the site of MM Ser, this will not truly be an optimal extraction in this case. The spectra extracted in this way were still far superior to a non-optimal extraction, however.

Wavelength calibration was interpolated relative to exposures of a $\mathrm{CuNe}+\mathrm{CuAr}$ lamp and checked against night sky lines. The rms scatter among the latter was $0.25 \AA$ in the blue and $0.14 \AA$ in the red, with no systematic offset in either case.

Flux calibration was applied relative to the spectrophotometric standard $\mathrm{BD}+33^{\circ} 2642$ (Oke 1990); this was also used to correct for Telluric absorption features. As an 0.8 arcsec slit was used significant uncertainty will be introduced in the flux calibration due to slit losses, especially as the slit was not aligned at the parallactic angle due to the need to follow the line of centres of the blended star. Consequently, the flux calibration of the spectra is only approximate.

The average extracted spectra (a composite of the two blended stars) is shown in Fig. 11 The presence of strong emission lines in the combined spectrum suggests that the brighter, bluer star is the optical counterpart to MM Ser as suggested by Wachter (1997). To confirm this we also used the spectral deblending method of Hynes (2002) to extract spectra of the two stars separately.

1 IRAF is distributed by the National Optical Astronomy Observatories, which are operated by the Association of Universities for Research in Astronomy, Inc., under cooperative agreement with the National Science Foundation.
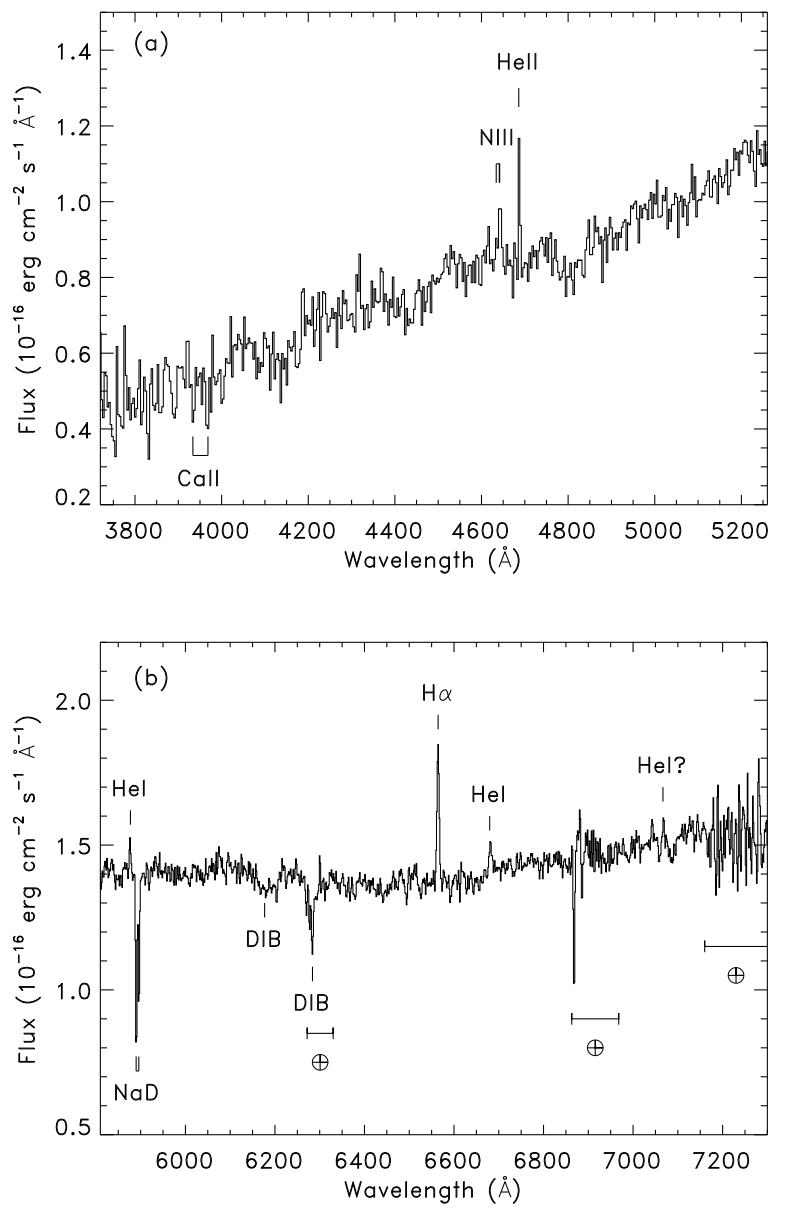

Figure 1. a) Combined blue spectrum of the two stars, binned to $3.5 \AA$ pixels. This will be dominated by MM Ser with the other star contributing less than 20 percent of the light. b) Combined red spectrum. Identified emission and interstellar absorption features are marked. Telluric correction has been applied, but regions which may be contaminated by residuals from this process are marked $\oplus$. The Telluric feature around $6300 \AA$ is much weaker than the longer wavelength features, so this region is probably not significantly contaminated.

\section{$2.2 \quad$ VLT Spectra}

Three further spectra were obtained with the FORS2 spectrograph at the Very Large Telescope (VLT) on 2002 May 18. The $1400 \mathrm{~V}$ grism was used with an MIT/Lincoln Labs mosaic CCD and an 0.7 arcsec slit. The seeing was around 1.2 arcsec. Further details are given in Table 1

Images were debiased and flatfielded using Starlink CCDPACK routines. As no suitable PSF template was available, and the contribution of the fainter star appeared very small, we only performed a single object 'optimal' extraction with IRAF as above. The images were marred by a number of bright spots, typically up to $20-30$ pixels across. These originate from enhanced dark current in regions that had been heavily saturated during earlier observations. We examined the saturated images to identify which regions of the spectrum might be affected. Fortunately, there were only a few mildly saturated regions coincident with the location of our 
Table 1. Log of spectroscopic observations of MM Ser.

\begin{tabular}{llllcccc}
\hline Date & Telescope & $\begin{array}{l}\text { Start } \\
\text { time (UT) }\end{array}$ & $\begin{array}{l}\text { End } \\
\text { time (UT) }\end{array}$ & $\begin{array}{c}\text { Number of } \\
\text { exposures }\end{array}$ & $\begin{array}{c}\text { Exp. } \\
\text { time }(\mathrm{s})\end{array}$ & $\begin{array}{c}\text { Wavelength } \\
\text { range }(\AA)\end{array}$ & $\begin{array}{c}\text { Resolution } \\
(\AA)\end{array}$ \\
2001 July 8 & WHT/ISIS & $22: 09$ & $23: 54$ & 4 & 1500 & $3600-5390$ & 1.9 \\
2001 July 8 & WHT/ISIS & $22: 09$ & $23: 54$ & 4 & 1500 & $5870-6660$ & 1.5 \\
2001 July 10 & WHT/ISIS & $21: 47$ & $22: 47$ & 2 & 1800 & $3600-5390$ & 1.9 \\
2001 July 10 & WHT/ISIS & $21: 47$ & $22: 48$ & 2 & 1800 & $5810-7300$ & 3.1 \\
2002 May 18 & VLT/FORS2 & $08: 29$ & $09: 25$ & 3 & 1100 & $4510-5810$ & 1.8 \\
\hline
\end{tabular}

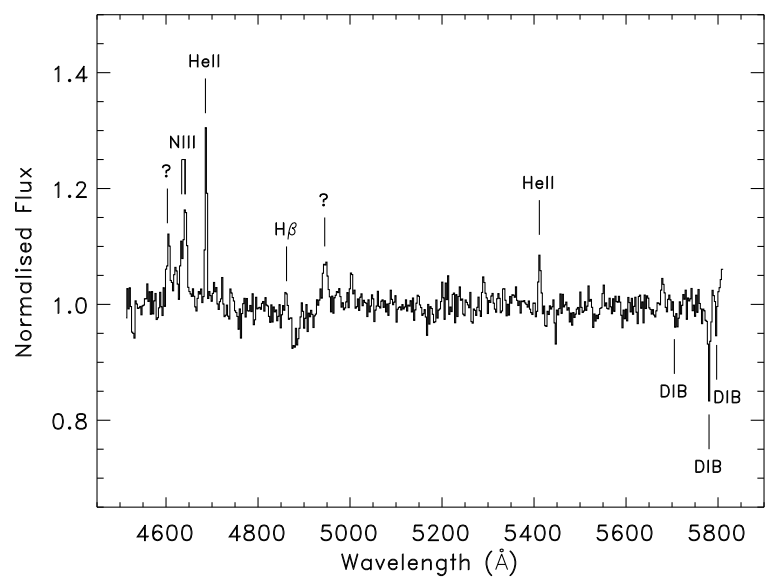

Figure 2. Average, normalised VLT spectrum of MM Ser. Identified features are marked. Unidentified features appear to be present at 4605 and $4945 \AA$. These are repeatable, and not obviously artifacts.

spectra, and none of these correspond to apparent features in the extracted one-dimensional spectra. We therefore believe that this problem does not compromise our dataset. Wavelength calibration was applied with respect to an arc image taken during the day. The wavelength was checked with respect to sky lines (mainly [O I] $5577 \AA$ ). There was an offset of $+0.21 \AA\left(13 \mathrm{~km} \mathrm{~s}^{-1}\right.$ at $\left.4686 \AA\right)$ which was corrected. This is within the expected flexure for FORS2 (Szeifert 2002). Given the large slit losses due to poor seeing, no flux calibration of this spectrum was attempted. The final average spectrum is shown in Fig. 2

\section{IDENTIFICATION OF THE COUNTERPART}

To separate the two stars we applied the deblending algorithm of Hynes (2002) to the July 10 red WHT spectra. These had the best spatial resolution and were well exposed, and were the only spectra suitable for this work. The stars were not fully resolved (Fig. 3), but were sufficiently separated that they could be identified and deblended. We defined the spatial profile with a base Voigt function, with width allowed to vary with wavelength, together with a wavelength independent numerical correction. The deblended spectra are shown in Fig. 4

It is clear that the emission features detected do originate from the bright star. $\mathrm{H} \alpha$ is particularly striking, but

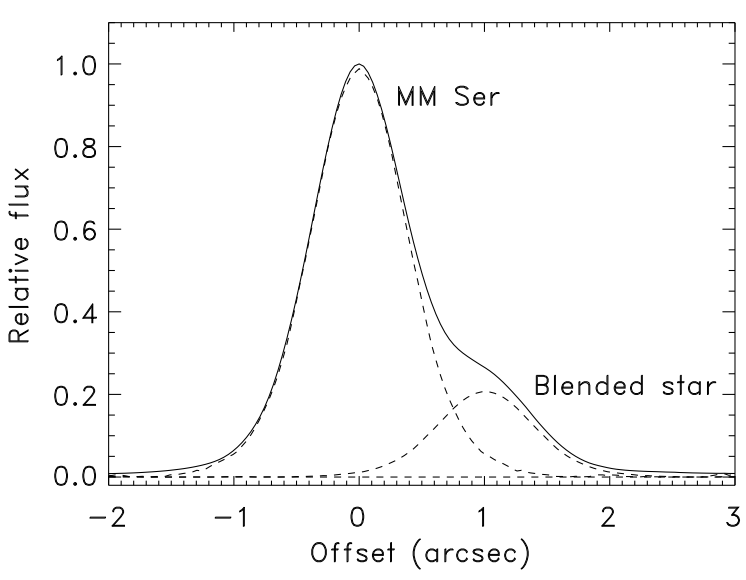

Figure 3. Average WHT red spatial profile showing MM Ser and the blended star. The solid line shows the data, oversampled and averaged over wavelength after removing the curvature of the spectrum. The dashed lines show the corresponding averages of the two modelled profiles.

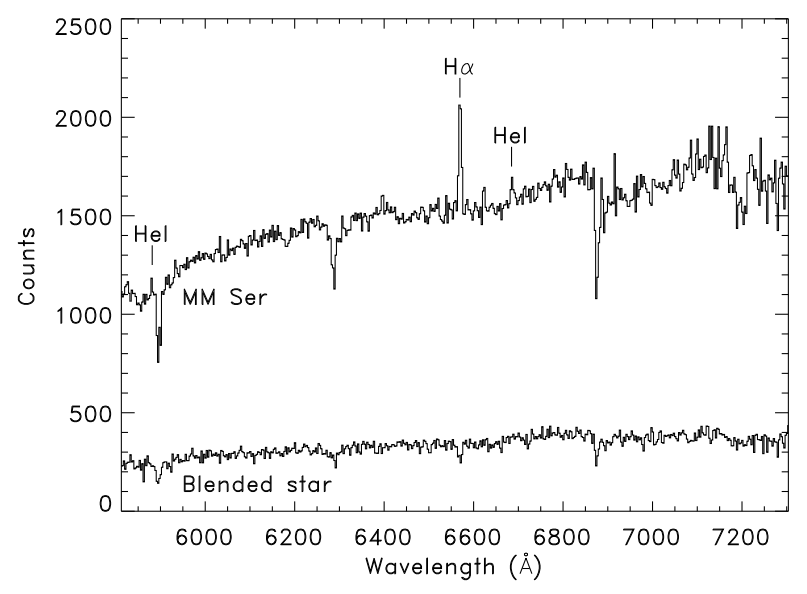

Figure 4. Average of the two deblended red spectra of MM Ser and the nearby star from 2001 July 10. It is clear that the emission lines, particularly $\mathrm{H} \alpha$, are associated with the brighter of the two stars. 
He I $6678 \AA$ is also recognisable. These features are clear in both individual spectra as well as in the average. This provides further confirmation of earlier suggestions (Wachter 1997; Silber 1998) that the brighter of the two stars, DSe in the terminology of Wachter (1997), is the LMXB.

Comparing the two components, at the epoch observed the fainter star contributes only about $15-20$ percent of the total light in the $6000-7000 \AA$ band. For comparison, the magnitudes given by Wachter (1997) correspond to contributions of 19 percent at $V$ and 25 percent at $R$.

\section{THE OPTICAL SPECTRUM OF MM Ser}

\subsection{Emission lines}

The only emission lines clearly detected in the WHT blue spectrum are He II $4686 \AA$ and the N III Bowen blend around $4640 \AA$; there are no strong Balmer emission lines in the blue. The Bowen blend contains at most weak $\mathrm{C}$ III emission. Moving to the red, the WHT spectrum is dominated by $\mathrm{H} \alpha$, with He I 5875 and $6678 \AA$ also present. The VLT spectrum partially overlaps the WHT blue-side, but also fills in coverage around $5500 \AA$. It shows the same blue lines, of N III and He II $4686 \AA$, with a similar relative strength. The He II $5411 \AA$ line is also clearly present. Weak $\mathrm{H} \beta$ emission does appear to be present, on the blue-side of a broad absorption trough. This is a ubiquitous feature in both black hole and neutron star LMXB spectra, e.g. Buxton \& Vennes (2003); see also Soria et al. (2000) and references therein for other black hole examples and Hynes et al. (2001) and Casares et al. (2003) for other neutron star cases. If it is indeed associated with $\mathrm{H} \beta$, the large redshift $\left(\sim 1000 \mathrm{~km} \mathrm{~s}^{-1}\right)$ remains unexplained. It is possible that the apparent redshift is only due to filling in of the blue absorption by emission, however. Such absorption troughs are usually attributed to the inner optically thick accretion disc (e.g. Soria et al. 2000), but a large redshift would not then be expected. Apparent features are seen at 4605 and $4945 \AA$. They do not appear to be artifacts, but there is no obvious identification and no strong features are seen in other LMXBs at these wavelengths. Given the inferior quality of the WHT spectra, they would probably be undetectable there. Properties of lines measured in all of the spectra are summarised in Table 2

All of the lines are remarkably narrow, as can clearly be seen in Fig. 5 where the line profiles in velocity space are collated. The FWHM listed in Table 2 are based on single Gaussian fits to the unbinned spectra. The contribution from the instrumental resolution has been corrected for, but is never dominant. For comparison the FWHM of He II in $4 \mathrm{U} 1822-371$ (Casares et al. 2003) is FWHM $750 \mathrm{~km} \mathrm{~s}^{-1}$, and the lines in $4 \mathrm{U} 1735-444$ and $4 \mathrm{U} 1636-536$ respectively have FWHMs of $660 \mathrm{~km} \mathrm{~s}^{-1}$ and $1220 \mathrm{~km} \mathrm{~s}^{-1}$ (Casares et al. in preparation). Such narrow emission lines are thus rather atypical for an LMXB. If they are disc lines, then the narrowness could indicate either a long period, and hence a large disc with a low outer velocity, or a low inclination, or a combination of both. Without fully resolving the profiles and confirming that they are disc lines, it is of limited value to model them. Applying some simple models to the He II $4686 \AA$ line, however, suggests that for typical neutron star LMXB parameters, if the period is less than 1 day then

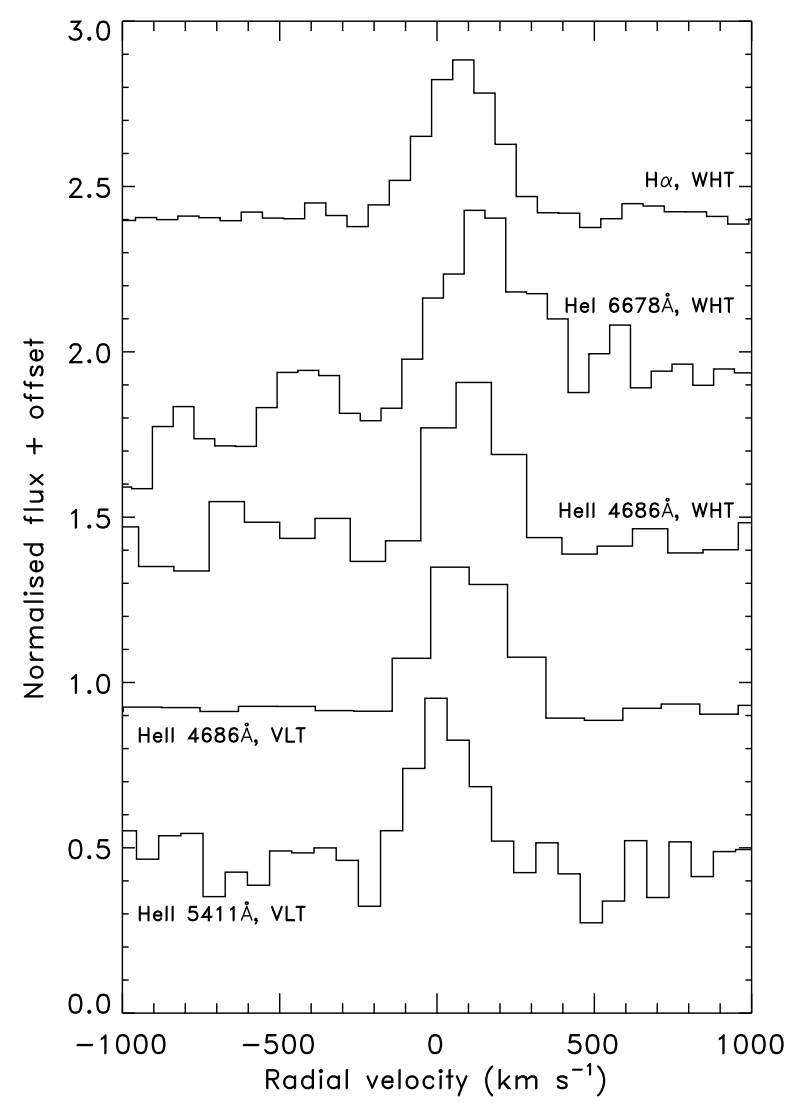

Figure 5. Line profiles of the main, identified, non-blended lines. All are rather narrow and appear single-peaked, at least to the limits of the resolution. Spectra have been binned to approximately one pixel per resolution element in each case. They have been continuum subtracted and normalised to the same total line flux, so that the shapes of the profiles can be compared.

$i \lesssim 20^{\circ}$. A longer period allows a somewhat higher inclination; for 10 days then $i \lesssim 45^{\circ}$. These are not very robust estimates, however, as when system parameters are known, apparent sub-Keplerian velocities are often inferred from disc line profiles (Marsh 1998). Alternatively the lines may not be dominated by the disc; they could come from the companion star or stream-impact point, or some other region (as in XTE J2123-058; Hynes et al. 2001), in which case the narrowness would be more natural. In this case, unless the inclination were low, we would expect the lines to move. There is clearly no large velocity difference between the two epochs, and the He II $4686 \AA$ velocities measured are consistent within errors, especially allowing for some uncertainty in the absolute velocities. The WHT spectra are individually too poor to search for shorter timescale radial velocity variations. The three VLT spectra are better suited. They are equally spaced and the $5577 \AA$ night sky line is stable to $\pm 0.01 \AA$ between them. We measure Gaussian fit velocities for He II $4686 \AA$ in the individual spectra of $105 \pm 13 \mathrm{~km} \mathrm{~s}^{-1}$, $111 \pm 10 \mathrm{~km} \mathrm{~s}^{-1}$, and $133 \pm 9 \mathrm{~km} \mathrm{~s}^{-1}$ respectively. These suggest a small drift (over about an hour), but this is not significant at the 90 percent confidence level and more observations would be needed to confirm any motion. 
Table 2. Measured line properties.

\begin{tabular}{lllll}
\hline Date & Line & $\begin{array}{l}\text { LSR Velocity } \\
\left(\mathrm{km} \mathrm{s}^{-1}\right)\end{array}$ & $\begin{array}{l}\text { EW } \\
(\AA)\end{array}$ & $\begin{array}{l}\text { FWHM } \\
\left(\mathrm{km} \mathrm{s}^{-1}\right)\end{array}$ \\
2001 July 8-10 & N III 4634,41,42 & - & $1.9 \pm 0.4$ & - \\
(WHT/ISIS) & He II 4686 & $+127 \pm 11$ & $2.1 \pm 0.2$ & $200 \pm 30$ \\
& H I 6562 $(\mathrm{H} \alpha)$ & $+90 \pm 6$ & $2.1 \pm 0.1$ & $210 \pm 20$ \\
& He I 6678 & $+165 \pm 40$ & $0.5 \pm 0.1$ & $280 \pm 80$ \\
2002 May 18 & N III 4634,41,42 & - & $1.6 \pm 0.4$ & - \\
(VLT/FORS2) & He II 4686 & $+112 \pm 6$ & $1.4 \pm 0.1$ & $240 \pm 20$ \\
& He II 5411 & $+42 \pm 16$ & $0.6 \pm 0.1$ & $320 \pm 40$ \\
\hline
\end{tabular}

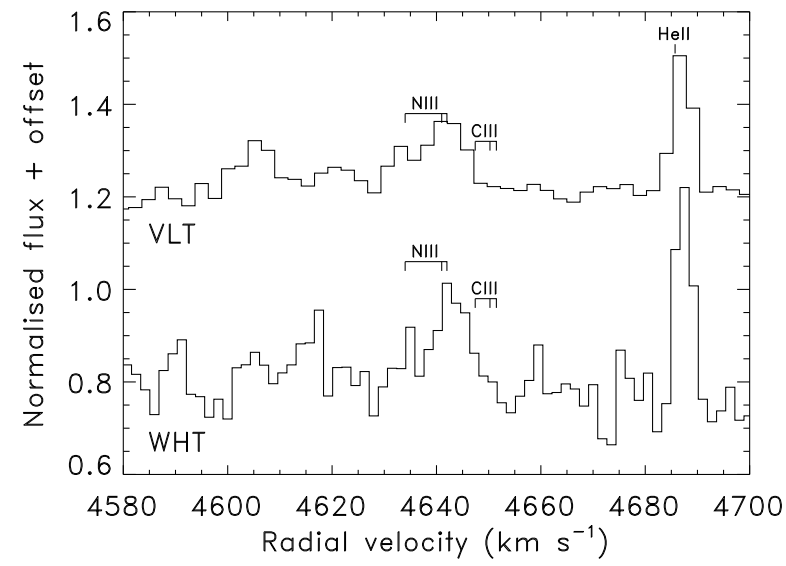

Figure 6. The spectral region containing the N III 4634/41/42 blend and He II $4686 \AA$. All annotations indicate the laboratory wavelength of expected lines. He II is clearly somewhat redshifted, and N III may be as well.

The region around the NIII lines and HeII $4686 \AA$ is expanded and compared in Fig. 6] The N III blend appears to show repeating structure with a strong peak corresponding to the $4641 / 42 \AA$ lines and a weaker peak for $4634 \AA$. There is no evidence for C III 4647/50/51 emission. That these components are resolved suggests that the individual components are narrow, like the isolated lines. The ratio of the Bowen and He II equivalent widths is $\sim 0.8$, typical of Galactic plane LMXBs which have values of 0.5-1.0 (Motch \& Pakull 1989).

\subsection{Interstellar features}

Our spectra show a number of interstellar features; NaD lines, diffuse interstellar bands (DIBs) and perhaps $\mathrm{Ca} \mathrm{H}$ and $\mathrm{K}$ lines. We can use these to constrain the amount of reddening toward MM Ser, although since these measures depend on measurements of equivalent widths, it is only valid to use measures from the deblended red spectrum. Shahbaz et al. (1996) suggested that the strong NaD feature in the spectrum of MM Ser indicated a G-type companion star. Since the spectrum shows strong diffuse interstellar bands (DIBs), we instead feel that it is more likely that the $\mathrm{NaD}$ absorption is also of interstellar origin. CaII $\mathrm{H}$ and $\mathrm{K}$ lines (3933,3968 $\AA$ ) are also marginally detected in the blue spec- trum, which should be dominated by the accretion light; hence these favour an interstellar origin. The interstellar absorption is therefore likely large.

The Na D doublet can be used as a reddening indicator, but is only reliable for small reddenings $(E(B-V) \lesssim 0.5)$, as it is prone to be saturated at larger values (Munari \& Zwitter 1997). We measure an EW for the NaD $5890 \AA$ line of $1.6 \pm 0.1 \AA$ from the deblended spectrum of DSe. This is larger than any values given by Munari \& Zwitter (1997), which saturate at a maximum value of about $1.2 \AA$. There is a spread in the saturation values, however, as it depends on the relative velocities of the clouds contributing to the absorption. A larger maximum NaD EW is seen when multiple resolved components are present as this partly circumvents saturation. Since all of the stars considered by Munari \& Zwitter (1997) are at $<3 \mathrm{kpc}$ and MM Ser has an estimated distance of $8.4 \mathrm{kpc}$ (Christian \& Swank 1997), it is likely that a larger EW is possible in the latter, since $\mathrm{NaD}$ absorption will be spread over a wider range of velocities and hence be less affected by saturation. We can still use the $\mathrm{NaD}$ strength as a lower limit for the reddening (if it is of interstellar origin), since the asymptotic linear relation between $\mathrm{EW}$ and $E(B-V)$ at low reddenings defines the limiting case of unsaturated absorption. The relation given by Munari \& Zwitter (1997) will then define a lower limit of of $E(B-V) \gtrsim 0.4$. We note that the ratio of the lines is also an indicator of reddening (Munari \& Zwitter 1997), since the NaD $5896 \AA$ line is intrinsically weaker and so saturates more slowly. At low optical depths the ratio is 2.0 , but this decreases to 1.1 as the reddening increases. We measure a ratio of $1.3 \pm 0.1$ suggesting that at least some components are saturated and hence that the reddening is high, significantly larger than the lower limit.

A relatively high reddening is supported by the strong DIBs; from the DIB at $6203 \AA$ we measure an EW of $270 \pm 130 \mathrm{~m} \AA$, implying $E(B-V)=0.8 \pm 0.4$ (Herbig 1975). The DIB at $6283 \AA$ may also be strong, but will not be reliable due to Telluric contamination. This value is consistent with the column density of $N_{\mathrm{H}}=0.5 \times 10^{21} \mathrm{~cm}^{-2}$ estimated by Christian \& Swank (1997), since the latter would imply $E(B-V)=0.6-1.1$ (e.g. Bohlin et al. 1978; Predehl \& Schmidtt 1995). We conclude that MM Ser is moderately reddened, with all indicators consistent with a reddening $E(B-V) \sim 0.6-1.1$. 


\section{CONCLUSIONS}

We have performed spectroscopy of MM Ser, the optical counterpart to Ser X-1. We resolve the two close components identified by Wachter (1997) and confirm earlier suggestions that the brighter of the two is an emission line source and hence the true counterpart. The spectra reveal emission lines of Hi, HeI, HeII, and NiII, as well as interstellar features. The emission lines are unusually narrow. Since all lines show similar widths, the most likely explanation seems that they are disc lines, but that the binary inclination is very low. Alternatively they could also originate from a more localised region such as the companion star or stream-impact point, but we would then require that the disc not dominate any of the optical lines. Further time-resolved observations would be needed to search for motion of the lines and discriminate between these possibilities. Higher resolution spectroscopy would also be beneficial to resolve the narrow line profiles better and study the substructure of the N III blend. Based on the strong interstellar lines, we have estimated a reddening consistent with estimates based on the X-ray derived neutral hydrogen column density of $E(B-V)=0.6-1.1$.

\section{ACKNOWLEDGEMENTS}

We are grateful to Carole Haswell for providing details of Silber (1998), and to Hilmar Duerbeck for permission to examine his proprietary VLT images. RIH and PAC acknowledge support from grant F/00-180/A from the Leverhulme Trust. RIH is currently funded from NASA through Hubble Fellowship grant \#HF-01150.01-A awarded by the Space Telescope Science Institute, which is operated by the Association of Universities for Research in Astronomy, Inc., for NASA, under contract NAS 5-26555. LvZ acknowledges the support of scholarships from the Vatican Observatory, the National Research Foundation (South Africa), the University of Cape Town, and the Overseas Research Studentship scheme (UK). DS acknowledges the support of a PPARC Postdoctoral Fellowship and a Smithsonian Astrophysical Observatory Clay Fellowship. The William Herschel Telescope is operated on the island of La Palma by the Isaac Newton Group in the Spanish Observatorio del Roque de los Muchachos of the Instituto de Astrofísica de Canarias. The Very Large Telescope is operated by the European Southern Observatory at Cerro Paranal in Chile. This research has made use of the SIMBAD database, operated at CDS, Strasbourg, France and the NASA Astrophysics Data System Abstract Service.

\section{REFERENCES}

Bohlin R. C., Savage B. D., Drake J. F., 1978, ApJ, 224, 132

Buxton M., Vennes S., 2003, MNRAS, 342, 105

Casares J., Steeghs D., Hynes R. I., Charles P. A., O'Brien K., 2003, ApJ, 590, 1041

Christian D. J., Swank J. H., 1997, ApJS, 109, 177

Cowley A. P., Hutchings J. B., Crampton D., 1988, ApJ, 333, 906

Davidsen A., 1975, IAU Circ. 2824

Doxsey R., 1975, IAU Circ. 2820

Friedman H., Byrom E., Chubb T., 1967, Science, 156, 374

Hackwell J. A., Grasdalen G. L., Gehrz R. D., Cominsky L., Lewin W. H. G., van Paradijs J., 1979, ApJ, 233, L115

Herbig, G. H., 1975, ApJ, 196, 129
Horne K., 1986, PASP, 98, 609

Hynes R. I., 2002, A\&A, 382, 752

Hynes R. I., Charles P. A., Haswell C. A., Casares J., Zurita C., Serra-Ricart M., 2001, MNRAS, 324, 180

Marsh, T. R. 1989, PASP, 101, 1032

Marsh, T., 1998, ASP Conf. Ser. 137: Wild Stars in the Old West, 236

Motch C., Pakull M. W., 1989, A\&A, 214, L1

Munari U., Zwitter T., 1997, A\&A, 318, 269

Oke J. B., 1990, AJ, 99, 1621

Predehl P., Schmitt J. H. M. M., 1995, A\&A, 293, 889

Shahbaz T., Smale A. P., Naylor T., Charles P. A., van Paradijs J., Hassall B. J. M., Callanan P., 1996, 282, 1437

Silber J., 1998, M.Sc. Thesis, University of Sussex

Soria R., Wu K., Hunstead R. W., 2000, ApJ, 539, 445

Steeghs D., Casares J., 2002, ApJ, 568, 273

Szeifert T. (ed.), 2002, FORS 1+2 User Manual, Issue 2.5, ESO

Thorstensen J. R., Charles P. A., Bowyer S., 1980, ApJ, 238, 964

Wachter S., 1997, ApJ, 490, 401 